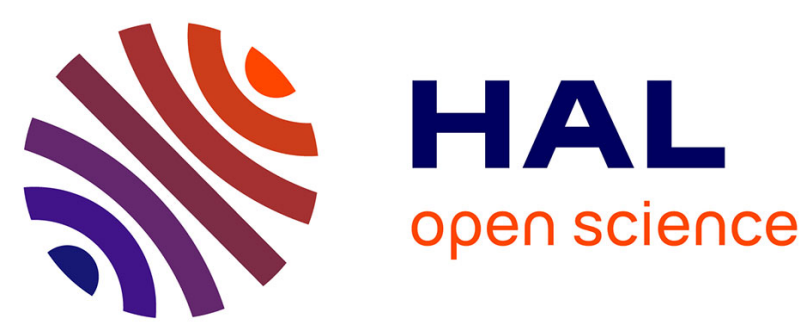

\title{
Il n'y a plus d'évaluation
}

Alain Vanier

\section{To cite this version:}

Alain Vanier. Il n'y a plus d'évaluation. Journal français de psychiatrie, 2007, Evaluation, 2 (29), pp.17 - 19. 10.3917/jfp.029.0017. hal-01523566

\section{HAL Id: hal-01523566 https://hal.science/hal-01523566}

Submitted on 6 Jun 2017

HAL is a multi-disciplinary open access archive for the deposit and dissemination of scientific research documents, whether they are published or not. The documents may come from teaching and research institutions in France or abroad, or from public or private research centers.
L'archive ouverte pluridisciplinaire HAL, est destinée au dépôt et à la diffusion de documents scientifiques de niveau recherche, publiés ou non, émanant des établissements d'enseignement et de recherche français ou étrangers, des laboratoires publics ou privés. 



\title{
Il n'y a plus d'évaluation ${ }^{1}$
}

\author{
Alain Vanier*
}

É valuer: nous ne cessons d'évaluer, de donner une valeur, de porter un jugement, ne serait-ce que sur notre pratique. Mais, plus radicalement, parce que notre relation au monde est réglée par des jugements de valeur qui l'ont fondée. Ainsi, dès le départ pour l'infans, lors des premières satisfactions, il y a un partage entre ce qui est bon et ce qui est mauvais : les saveurs des premières satisfactions orales font accepter ce qui est considéré comme bon et rejeter ce qui est mauvais. La saveur est un savoir, et il y a un nouage initial entre savoir et jouissance. Je pense à ce petit garçon de quelques mois qui ne mangeait que les aliments salés chez sa nourrice et les produits sucrés chez sesparents.

Nous vivons dans l'ère du jugement ; la difficulté est que cette notion a pris un tour spécifique dans le monde contemporain. Il y a diverses manières de situer ce tournant. Ainsi, Walter Benjamin, dans son essai sur le langage humain, laissait paraître une certaine nostalgie puisque, pour lui, le monde d'aujourd'hui, celui de l'ère du jugement-Urteil-manifeste une forme dégradée du langage divin où mot et chose correspondaient où la langue était nomination. Elle n'a plus aujourd'hui qu'une valeur d'usage, un statut d'outil. Benjamin propose un mythe d'origine du langage et de son mode contemporain de dégradation. Quelles sont les valeurs qui fondent les évaluations actuelles et quels rapports ontelles au jugement comme position d'un sujet ? Quoi qu'il en soit, elles produisent des valeurs qui orientent le lien social et définissent peu à peu une éthique. Elles sont liées au type de valeur et de

\footnotetext{
* Psychanalyste, professeur à l'université de Paris 7
}

norme qu'a produit l'irruption de la science et de ses effets dans notre monde, en premier lieu un effet d'homogénéisation, d'équivalence généralisée. La valeur de l'expérience décline, comme ce qui la fondait. Hannah Arendt pouvait écrire: «Si l'on compare le monde moderne avec celui du passé, la perte d'expérience humaine que comporte cette évolution est extrêmement frappante [... ]. La pensée elle-même, en devenant "calcul des conséquences", est devenue une fonction du cerveau, et logiquement on s'aperçoit que les machines électroniques remplissent cette fonction beaucoup mieux que nous. » Elle ajoute : «Dans le même temps, nous nous sommes montrés assez ingénieux pour trouver les moyens de soulager la peine de vivre, à tel point qu'il n'est plus utopique de songer à éliminer le travail du nombre des activités humaines. Car dès à présent, le mot travail est trop noble, trop ambitieux, pour désigner ce que nous faisons ou croyons faire dans le monde où nous sommes. Le dernier stade de la société de travail, la société d'employés, exige de ses membres un pur fonctionnement automatique, comme si la vie individuelle était réellement submergée par le processus global de la vie de l'espèce, comme si la seule décision encore requise de l'individu était de lâcher, pour ainsi dire, d'abandonner son

\section{La valeur}

\section{de l'expérience}

décline,

\section{comme ce qui}

la fondait.

1. Reprise d'une communication au séminaire de printemps, "L'évaluation : nécessite scientifique ou servitude volontaire? », organisé par l'AFPEP, le Collège de psychiatrie et le Journal français de psychiatrie à Paris, les 1er-2 avril 2006.

2. Hannah Arendt (1958),

Condition de l'homme moderne, trad.G.Fradier,Paris, CalmannLévy, 1961 et 1963. individualité, sa peine et son inquiétude de vivre encore individuellement senties, et d'acquiescer à un type de comportement, hébété, "tranquillisé" et fonctionnel. Ce qu'il y a de fâcheux dans les théories modernes du comportement, ce n'est pas qu'elles sont fausses, c'est qu'elles peuvent devenir vraies, c'est qu'elles sont, en fait, la meilleure mise en concept possible de certaines tendances évidentes de la société moderne 2 . » Chacun est ainsi sommé de prendre sa place dans l'immense machine de production et on peut évoquer le grand théâtre de l'Oklahoma dont parle Kafka à la fin de L'Amérique, qui met chacun à sa place.

Benjamin parlait aussi du déclin de la valeur de l'expérience, sous l'angle de la narration. Ce désintérêt, sensible après la Première Guerre mondiale, avait été aussi relevé par Freud. Il s'agit d'une perte de l'expérience comme transmissible et d'une perte de l'expérience comme valeur. La Première Guerre mondiale a été la fin del'expérience de la guerre au sens qu'elle avait jusque-là, supposant un certain nombre de valeurs. Benjamin notait déjà que la guerre moderne disqualifie d'emblée les règles qu'elle édicte, comme le principe de protection des populations civiles. Il s'est agi pour la première fois d'une guerre de maté- 
riel et non d'une guerre humaine. Là où le corps à corps avec l'ennemi et les valeurs de l'héroïsme prévalaient, Freud comme Benjamin notent que cette guerre n'implique plus aucune reconnaissance mutuelle dans le combat, qu'elle rend obsolètes les valeurs de l'héroïsme, et qu'elle engage aussi un changement du statut du corps. Il n'est plus un lieu de reconnaissance dans le combat mais un simple élément matériel. «Les attaques au gaz [...] promettent de donner à la guerre future un visage qui abolira définitivement les catégories guerrières au profit des catégories sportives, qui ôtera aux opérations tout caractère militaire et les rangera entièrement dans la logique du record 3. » Si on pense aux conceptions stratégiques et aux manières de faire la guerre jusqu'au XVIII siècle, on s'aperçoit qu'il s'agissait d'épargner les hommes, la bataille souvent n'était pas livrée : la prise d'une position signifiait une victoire anticipée et pouvait prévenir une bataille. Pour Benjamin, le strict nombre de morts, civils ou militaires, indistincts mais surtout civils, règle maintenant l'issue. Pour autant, attention à notre passéisme, à notre nostalgie. En effet, le texte de Benjamin est une réponse à un livre d'Ernst Jünger, intitulé Krieg und Krieger, qui déplore ce nouveau visage de la guerre et se propose de restaurer les valeurs perdues.

Le corps a changé de statut et la médecine est sans doute le lieu exemplaire de cette mutation. Ainsi la greffe a fait du corps du prochain un réservoir potentiel d'organes, a pu dire Jean-Michel Zucker. Le corps, c'est-à-dire ce à quoi est condamné le sujet (et non à sa conscience), soulignait Lacan.

Valeur vient de valere, qui veut dire être bien portant, fort, être puissant et donc valoir. Or être bien portant est devenu une affaire d'Etat. Sur ce changement du corps et sur l'engagement de l'État dans le problème de la santé, il est impossible de ne pas évoquer le travail de Michel Foucault 4 . Pour Foucault, la médecine moderne a pour fondement une certaine technologie du corps social et son lien avec le capitalisme ne se manifeste pas par le passage d'une médecine collective à une médecine privée, comme on le croit trop souvent, mais par l'inverse. Il faut néanmoins distinguer cette position de celle d'Henri Ellenberger, qui indiquait comment la maladie était conçue autrefois 5 . Pour lui, les techniques de guérison primitives considéraient que la maladie était une rupture de l'unité du vivant, mais aussi du corps social. Le lien entre maladie et groupe social faisait que le traite-

\section{Le corps}

a changé

\section{de statut}

\section{et la médecine}

est sans doute

le lieu

exemplaire

de cette

mutation.

3. Walter Benjamin (1930),

«Théories du fascisme

allemand », trad. P. Rusch, dans Euvres, t. II, Paris, Gallimard, 2000.

4. Sur la question du ou de la biopolitique, on consultera en particulier M. Foucault, Dits et écrits, t. III et IV (ou t. II coll. « Quarto »), Paris, Gallimard, 1974.

5. H. F. Ellenberger, Histoire de la découverte de l'inconscient, trad. J. Feisthauer, Paris, Fayard, 1994 1re éd. Simep, 1974

6. G. Canguilhem, Le normal et le pathologique, Paris, PUF, 1966 7. D. Lecourt (sous la direction de), article «Normes», dans Dictionnaire de la pensée médicale, Paris, PUF, 2004. ment était généralement public et collectif. Avec la modernité, autre chose est en jeu : il s'agit de socialiser un objet, le corps, en fonction de la force productive, c'est-à-dire de la force de travail. On se souviendra de la fonction de l'oisiveté comme critère relevé par Foucault lors de la création de l'hôpital général. Pour le capitalisme, «le contrôle des individus se fait par le corps », et le corps est une réalité biopolitique, la médecine une stratégie biopolitique. Une médecine d'État, une medizinische Polizei, se met en place, une médecine sociale qui introduit l'idée, nouvelle au XVIIII siècle, d'hygiène publique, dans le même temps où paraît le premier programme d'hygiène des populations. La biopolitique traite une population à partir de savoirs et de techniques spécifiques, elle est « la manière dont on a essayé depuis le XVIIII siècle de rationaliser les problèmes posés à la pratique gouvernementale par les phénomènes propres à un ensemble de vivants constitués en population ». Ainsi, « la vie est devenue maintenant, à partir du XVIII siècle, un objet de pouvoir : la vie et le corps».

Sous un autre angle, Canguilhem a étudié le développement de la médecine au XIXe siècle, et son appui nouveau sur la biologie et la physiologie - la découverte des microbes, etc. -, ainsi que le développement de la notion d'hygiène publique 6 . Il parle, à ce propos, de " séisme épistémologique », et Lacan de l'accroissement d'un « hiatus épistémo-somatique » entre le corps du physiologiste et le corps de lajouissance.

La conception actuelle de la médecine, organisée autour de la norme biologique, identifiée à un fait objectif supposé définir la santé, ne s'accorde-t-elle pas à merveille avec la conception de la norme sociale comme réalité intangible des sciences naturelles ? questionne Dominique Lecourt 7. En effet, l'augmentation de la confiance dans la médecine s'accompagne aujourd'hui d'un accroissement de la peur des maladies. La médecine terrifie, comme l'Église autrefois, et renvoie chacun àluimême en l'enjoignant de se normaliser : se rendre conforme à une «norme » scientifiquement définie. Le risque de l'écart épouvante : " Ordre moral et ordre humain échangent leurs valeurs. »

Aujourd'hui, le clinicien disparaît - constat banal - et la clinique avec lui. Le médecin, de plus en plus, prend le point de vue du physiologiste. Mais normal et pathologique du point de vue du 
physiologiste coïncident-ils avec l'opposition vécue par l'individu entre santé et maladie ? Cette remarque de Dominique Lecourt évoque bien sûr la position de Canguilhem, lequel définit la santé comme la modalité suivant laquelle un organisme invente sans cesse de nouvelles normes. La vie est un processus irréversible - il n'y a rien de plus mauvais pour la santé que de vieillir, disait Raymond Devos. Il ajoutait : «J'ai décidé d'arrêter ! Mais de temps en temps, je me laisse un peu aller et je prends un petit coup de vieux. » N'est-ce pas ce qui nous arrive dans ce monde où la santé est présentée comme conformité à une norme « naturelle » ? La vie est devenue sacrée. Pour Hannah Arendt, elle est le souverain bien d'aujourd'hui. Pour Walter Benjamin, ce caractère sacré de la vie est récent ; il correspond à l'égarement de la pensée occidentale qui cherche ainsi à récupérer le sacré qu'elle a perdu.

On saisit bien la difficulté que présente la psychanalyse pour qui l'inconscient est source de normes, le statut de la valeur tenant à un objet éminemment problématique, que Lacan repérait dans Marx comme plus-value. Le sujet entre dans le lien social par un renoncement à la jouissance, et le lien social s'organise en retour comme modalité de canalisation de la jouissance. En effet, l'entrée en jeu du signifiant mortifie le corps et implique un clivage entre corps et jouissance. $\mathrm{Ce}$ renoncement fonde notre possibilité de savoir, à ceci près que nous sommes dans le temps d'une mutation du régime du savoir liée aux effets de la science. La perte du lien du savoir à la vérité du sujet est patente, la science, en effet, ne se soucie aucunement de cette vérité. Elle l'a réduite comme dans la logique formelle aux valeurs de vérité. La vérité ici est vidée, transformée en instrument du savoir scientifique. La vérité du sujet n'est que supposée et «ne peut être que mi-dite». D'autant que « dans le monde du marché, le savoir est devenu une marchandise ». On sait qu'autour des années 1968, la création à l'université des « unités de valeur » manifestait, pour Lacan, une évolution de l'université moderne et du savoir marchandisé.

À partir d'un certain jour, celui du début du capitalisme, «le plus-de-jouir se compte, se comptabilise, se totalise. Là, commence ce que l'on appelle accumulation du capital ». Jusque-là et fondamentalement, on ne pouvait joindre le sujet du signifiant à cette jouissance qui n'est plus. Or, le traitement du plus-de-jouir comme plus-value-qui s'adjoint au capital - l'impuissance de ce lien entre le sujet divisé et le plus-de-jouir se vide. On peut lire ces affirmations de Lacan comme une anticipation de ce qu'il écrira plus tard comme discours du capitaliste. Nous sommes entrés dans un monde qui est le monde de la promesse, promesse de récupére cette jouissance perdue. C'est un paradoxe, car cette jouissance est perdue du fait de l'entrée du suje dans le langage, et le sujet n'a dès lors à sa disposition que le langage pour pouvoir la récupérer. Autrefois, cette question appartenait au domaine de la foi.

En même temps, « le signifiant-maître n'en apparaît que plus inattaquable, justement dans son impossibilité. Où est-il ? Comment le nommer?». Il est d'autant plus inébranlable que «sa présence marque, occupe, bouchecequ'il peut en être dela vérité ». Il n'est aujourd'hui même plus incarné, c'est une entité vague, l'État, qui laisse apparaître de plus en plus son impuissance, et ce qu'il s'agit de protéger, c'est l'État lui-même. Tout ceci s'ac- compagne d'une montée des peurs, car, en effet, la vie est devenue sacrée. L'invisibilité de cette place du signfiant-maître est de plus en plus mal dissimulée et elle n'est pas sans rapport avec l'augmentation des innombrables théories du complot qui visent à fournir une garantie à l'Autre, à nommer cet Autre jouisseur. Aujourd'hui, cette vérité exclue revient sur un mode délirant. Les psychiatres auraient peut-être intérêt à se souvenir de cette notion aujourd'hui désuète de «psychose de quartier»

Cette vérité, l'hystérique s'en était fait l'emblème adressé au prêtre, puis au médecin, ce que vérifie l'histoire de la démonologie et de la psychiatrie, de Jean Bodin à Charcot. De cette place, le médecin cherche à se démettre de plus en plus, ce qui conduisait Lacan à se définir lui, l'analyste, comme le missionnaire du médecin. Mais il n'y a, dans le monde moderne, qu'une « vérité sociale moyenne », celle des statistiques et de l'évaluation contemporaine. Elle est bien différente de la façon dont chacun souffre dans son rapportàlajouissance, et la vague des psychothérapies adaptatives est là pour écraser ce qui se manifeste comme vérité.

Mais notre façon de typifier notre clinique risque d'être souvent une façon de masquer sa butée permanente, qui constitue son réel. Kraepelin aussi bien soulignait qu'il n'y a pas de cas purcorrespondant aux catégories nosographiques, tout comme Bercherie, plus près de nous, constatait l'échec de la grande clinique dans les années 1930 par la quasi-définition d'une catégorie par patient. Lacan, dans une interview de 1974, pouvait dire : « D'abord, rejetons cet homme moyen qui, lui, pour commencer, n'existe pas, c'est seulement une fiction statistique, il existe des individus et c'est tout. Quand j'entends parler de l'homme de la rue, de sondages, de phénomènes de masse ou de choses semblables, je pense à tous les patients que j'ai vu passer sur le divan de mon cabinet en quarante années d'écoute. Il n'y en a pas un qui soit de quelque façon semblable à l'autre, pas un avec les mêmes phobies, les mêmes angoisses, la même façon de raconter, la même peur de ne pas comprendre. L'homme moyen, qui est-ce, moi, vous, mon concierge, le président de la République ? »'érosion de la singularité est relevée aussi à un autre niveau par Walter Benjamin quand il évoque la perte de l'aura, ce qui conserve son unicité à une œuvre, liée à sa valeur cultuelle, qui disparaît dans la reproduction mécanisée. Il hésitera, dans les réécritures suc-

\section{La conception}

\section{actuelle}

de la médecine,

organisée

autour

delanorme

biologique,

identifiée

à un fait

objectif

supposé définir

la santé,

ne s'accorde-

t-elle pas

à merveille

avec

la conception

de la norme

sociale

comme réalité

intangible

des sciences

naturelles? cessives dece texte, entre une position nostalgique - regretter la disparition de l'aura et tenter de la restaurer - et puis, au contraire, s'en réjouir en espérant ainsi, à partir de cette destruction ultime, un franchissement. Ces oscillations sont fécondes et je crois que nous aurions tort d'épouser, comme on le voit trop souvent dans le champ psychanalytique, la radicalité d'une position ou d'une autre. De ces oscillations sur le sens de la modernité, la psychanalyse est née. Alors, vivons-nous le divorce à venirde la psychiatrie et de la psychanalyse, après quelques décennies de noce, dans le malentendu le plus total? Ne doit-on pas, comme certains le pensent, nous retirer des institutions, de la psychiatrie commede l'université, pour conserver la chose pure de la psychanalyse, parce que le capitalisme néolibéral entraîne une accélération de l'évaluation économique de toutes les pratiques en les réduisant au marché ? À un certain niveau, la psychanalyse est inévaluable. On peut évoquer le dispositif d'école de Lacan, où il proposait que l'expérience de chacun soit ainsi évaluée par les autres. Est-ce encore possible dès l'instant où la psychiatrie et la psychothérapie sont devenues affaires d'État? Il n'y a plus d'évaluation, seulement une réduction de valeurs : «Aujourd'hui, l'économie fonde la valeur », indiquait Lacan, elle devient le seul étalon objectif qui fonde le décidable. Sans doute conviendra-t-il de cultiver les marges, de constituer des îlots de résistance, mais cela ne suffira pas. $\mathrm{Ne}$ soyons pas nostalgiques du passé ; il reste quelque chose à créer : «Une pratique sans valeur, voici ce qu'il s'agirait pour nous d'inventer. $>$ 